\title{
Significados sobre el arte del cuidado desde la perspectiva de los estudiantes de Licenciatura en Enfermería
}

\section{Meanings of art of care from the perspective of bachelor's in nursing students}

\section{Significados sobre a arte do cuidado desde a perspectiva dos estudantes de Licenciatura em Enfermagem}

\author{
N. Ferro-Rivera ${ }^{\mathrm{a}^{*}}$, S. Rodríguez-Jiménez ${ }^{\mathrm{b}}$, M. Cárdenas-Jiménez ${ }^{\mathrm{b}}$ \\ a División de Estudios Profesionales, Escuela Nacional de Enfermería y Obstetricia, Universidad Nacional Autó- \\ noma de México, Ciudad de México

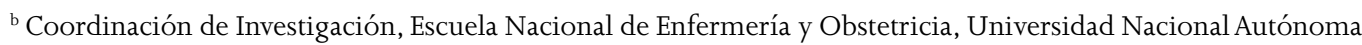 \\ de México, Ciudad de México
}

Recibido: 1 febrero 2018

Aceptado: 22 agosto 2018

\section{Resumen}

Objetivo: Comprender los significados sobre el arte del cuidar desde la perspectiva de los estudiantes de la Licenciatura en Enfermería de la ENEO-UNAM en el marco del enfoque humanístico que construyen durante su formación.

Metodología: Estudio cualitativo fenomenológico-hermenéutico desde la óptica de Heidegger y Gadamer, respectivamente, mediante la técnica de grupo focal, realizado con estudiantes de $2^{\circ}, 6^{\circ}$ y $8^{\circ}$ semestre de la Licenciatura de Enfermería de la ENEO-UNAM, tras obtener el consentimiento informado, se inició el trabajo grupal con una guía de preguntas generadoras.

Resultados: Se identificaron cinco categorías generales y diecisiete subcategorías; las primeras son: 1) Autopercepción de la enfermería, 2) Arte del cuidado, 3) Interacción enfermera-persona, 4) Conducta profesional y 5) Espiritualidad vs. Religiosidad, estas denotan los significados que le asignan los estudiantes al cuidado y al arte del cuidado en su proceso formativo.

Conclusiones: Para los estudiantes el arte del cuidado significa desarrollo de la sensibilidad, creatividad en el cuidado a la salud, autoconocimiento, reconocimiento del otro en una relación de alteridad, respeto a la dignidad de la persona, desarrollo de la espiritualidad en el contexto de la conducta profesional. Se 
destaca que la formación humanística se centra en cualificar al cuidado como holístico, con extensión a la familia, donde se reconocen los sentimientos implicados; hacen énfasis en el desarrollo de la identidad profesional-personal, como componente esencial de la enfermería.

Palabras clave: Cuidado; arte; estudiantes de enfermería; fenomenología; hermenéutica; México.

\section{Abstract}

Objective: To understand the meanings of the art of care from the perspective of students of bachelor's in nursing of ENEO-UNAM within the framework of their own formation's humanistic focus.

Methodology: This is a qualitative phenomenological-hermeneutical study based on Heidegger and Gadamer optics using the focus groups technique with nursing students from the 2nd, 6th, and 8th semesters. The corresponding informed consents were obtained and a questions guide was followed.

Results: Five general categories with seventeen sub-categories were identified: 1) self-perception of nursing; 2) the art of care; 3) nurses-persons interaction; 4) professional conduct; and 5) spirituality vs religiosity.

Conclusions: For students, the art of care means the development of sensitivity, creativity of care, self-appraisal, acknowledgement of the other as a unique person, respect for the dignity of the person, and spirituality within professional behavior. It was also highlighted that the humanistic formation is focused on regarding healthcare as an holistic process which encompasses the family, where, feelings are made conscious, and where the development of the personal-professional identity is an essential component.

Keywords: Care; art; students, nursing; phenomenology; hermeneutics; Mexico.

\section{Resumo}

Objetivo: Compreender os significados sobre a arte do cuidar desde a perspectiva dos estudantes da Licenciatura em Enfermagem da ENEO-UNAM, no marco do enfoque humanístico que constroem durante sua formação.

Metodologia: Estudo qualitativo fenomenológico-hermenêutico desde a óptica de Heidegger e Gadamer, respectivamente, mediante a técnica de grupo focal, realizado com estudantes de $2^{\circ}, 6^{\circ}$ e $8^{\circ}$ semestre da Licenciatura de Enfermagem da ENEO-UNAM, após obter o consentimento expresso, iniciou-se o trabalho grupal com uma guia de perguntas geradoras.

Resultados: Identificaram-se cinco categorias gerais e dezessete subcategorias; as primeiras são: 1) Autopercepção da enfermagem, 2) Arte do cuidado, 3) Interação enfermeira-pessoa, 4) Conduta profissional e 5) Espiritualidade vs. Religiosidade, estas denotam os significados que lhe assignam os estudantes ao cuidado e à arte do cuidado em seu processo formativo.

Conclusões: Para os estudantes a arte do cuidado significa desenvolvimento da sensibilidade, criatividade no cuidado à saúde, autoconhecimento, reconhecimento do outro em uma relação de alteridade, respeito à dignidade da pessoa, desenvolvimento da espiritualidade no contexto da conduta profissional. Destaca-se que a formação humanística centra-se em qualificar ao cuidado como holístico, com extensão à família, onde se reconhecem os sentimentos envolvidos; fazem ênfase no desenvolvimento da identidade profissional-pessoal, como componente essencial da enfermagem.

Palavras chave: Cuidado; arte; estudantes de enfermagem; fenomenologia; hermenêutica; México.

\section{Introducción}

La Escuela Nacional de Enfermería y Obstetricia (ENEO) UNAM, forma licenciados en Enfermería a través de un plan de estudio estructurado en tres ciclos de formación profesional: Fundamentos de Cuidado de Enfermería, que comprende el 1ro y 2do semestre; Cuidado en el Ciclo Vital Humano, incluye del 3er al 6to semestre y por último el Cuidado en la Salud Colectiva, constituido por el 7 mo y 8vo semestre; la organización de las asignaturas se da a través de las siguientes dimensiones: social, teórico-metodológica y filosófica-histórica. 
Las asignaturas que forman parte de la dimensión filosófica-histórica de interés para el estudio son: Historia Universal del Cuidado, Filosofía y Enfermería, Historia de la Enfermería en México, Epistemología y Enfermería, Ética Profesional y Legislación de la Enfermería, ubicadas en el primero y segundo ciclo de formación.

El plan curricular es el contexto de esta investigación, ya que en el perfil de egreso de la dimensión filosófica-histórica, entre otras, pretende que el egresado podrá ejercer una práctica basada en la ética y estética del cuidado, en la que reconoce a la persona como un ser holístico capaz de ejercer el autocuidado a la salud en pleno reconocimiento a los derechos humanos y sociales y demostrar habilidades para la interacción enfermera(o)-persona, con sentido humanístico a través de la acción comunicativa ${ }^{1}$.

El plan curricular plantea una formación profesional integral, esta se interpreta como la síntesis de una preparación sólida científico-técnica y laboral, integrada a una decidida formación humanística, caracterizada por el desarrollo de valores éticos, morales y culturales, con un alto nivel de compromiso social que garantice su aporte continuado al desarrollo global desde su profesión. Esto implica que la formación deberá verse como un proceso complejo y de continua reflexión en el entorno universitario ${ }^{2}$.

En enfermería, el proceso formativo es humanístico, pues se desarrolla en un acto de comprender y tratar al ser humano como persona ${ }^{2}$, desde una perspectiva holística. Wiedenbach referida por Pardo ${ }^{3}$, define la influencia humanística como la individualización de la persona, sus experiencias y el reconocimiento de la percepción ante el momento de salud o enfermedad que vive, es decir, centra el cuidado en el sujeto de estudio, el ser persona.

El proceso de cuidado lleva implícito el arte como una forma de expresión intencionada, según Orem, esto ocurre cuando la enfermera a través de su creatividad y estilo en el diseño de cuidados efectivos, satisface las necesidades humanas ${ }^{4}$, lo que implica capacidad empática, tener la habilidad de participar en la experiencia del otro, para percibir la expresión de los sentimientos a través de la escucha, mirada atenta y contacto físico 5 .

Por lo anterior, la formación en enfermería no sólo debe ser el dominio de los conocimientos científicos y tecnológicos, sino también la búsqueda del sentido humanístico, el desarrollo de la sensibilidad, la comunicación significativa, clarificación de las necesidades y sentimientos de la persona. Por ello, la tarea de la educación es humanizar, es decir poner a los estudiantes en contacto con las personas y los valores que ello representa ${ }^{6}$.

Para considerar esta formación como humanística, Garavito ${ }^{7}$ propone dimensiones que de manera conjunta expresan las potencialidades fundamentales que el estudiante debe desarrollar, estas son: ética, espiritual, cognitiva, sociopolítica, afectiva, comunicativa y estética, estas en conjunto le permiten adquirir los conocimientos, habilidades, aptitudes y actitudes para desarrollarse como un profesional con sentido humanístico integral.

Al realizar una búsqueda sistemática sobre la formación en el arte del cuidado, con esta perspectiva humanística, se encontraron estudios que se enfocan a un área específica de las especialidades médicas tales como: pediatría u oncología, entre otros; por ejemplo el de Parra et $\mathrm{al}^{8}$, quienes realizan su estudio en pacientes con cáncer, refieren que el contacto físico fortalece al individuo y permite a la persona generar confianza y disposición de ayuda en el cuidado; otro estudio en esta misma área es el de Falcão et $\mathrm{al}^{9}$ donde se aborda la producción, expresiones y significados derivados de la interacción entre pacientes y estudiantes, éstos dicen sentir gratificación en el cuidado, pues exploran su creatividad y ayuda a los demás con una técnica de expresión artística, en el cuidado a la persona.

Muñoz-Pino ${ }^{10}$ en su investigación relativa a las experiencias de los estudiantes de enfermería en su primer encuentro con pacientes en etapa terminal, refieren que para los alumnos conocer las emociones, sentimientos y sensaciones de la persona cuidada permite reconocer su situación y asimilarla, con el fin de disminuir la ansiedad y el temor. En el estudio de Magalhães, et $\mathrm{al}^{11}$ por su parte, los estudiantes entienden la dimensión espiritual como el apoyo, seguridad, confianza, orientación cuando la persona está angustiada y se busca dar consuelo, en el cuidado de enfermería. 
Por lo anterior, el objetivo de este estudio es comprender los significados sobre el arte del cuidar, desde la perspectiva de los estudiantes de la Licenciatura en Enfermería de la ENEO-UNAM, en el marco del enfoque humanístico que construyen durante su formación.

\section{Metodología}

Se trata de un estudio cualitativo, de corte fenomenológico-hermenéutico, busca la comprensión de los significados de los estudiantes, al arte del cuidado a través de las experiencias vividas durante su práctica y los conocimientos humanísticos del cuidado.

Este estudio se basa en primer término en la fenomenología de Heidegger, la que permite comprender las vivencias de la conciencia como objeto de estudio, son fenómenos que se descubren cuando existe una preocupación por ser develados; este método se divide en tres partes: destrucción, reconocimiento e identificación de tendencias, pensamientos iniciales frente al fenómeno, ya sean positivos o negativos; reducción, escuchar lo que se entiende del fenómeno y no ha sido expresado antes y por último, construcción, se espera lograr la compresión del fenómeno descrito en un proceso de pre-concepción hermenéutica ${ }^{12}$.

La hermenéutica por su parte permite la comprensión según Gadamer, que sirve de base a las teorías y metodologías para la interpretación de textos ${ }^{13}$, se busca a partir del diálogo la integración del análisis, conocimiento y pensamiento sobre la concepción de la experiencia de un fenómeno; por ello la fenomenología hermenéutica, transforma una comprensión distorsionada u oculta del ser, en una comprensión del ser en sí mismo.

La obtención de la información se realizó a través de la técnica "grupo focal”, se desarrolló a través de preguntas generadoras que orientó el trabajo de los grupos. Para integrar a los grupos, se seleccionaron aquellos estudiantes que cumplieran con los criterios de inclusión, estos son: estar inscrito en $2^{\circ}, 6^{\circ}$ u $8^{\circ}$ semestre, haber aprobado la asignatura de Filosofía y Enfermería, Epistemología y Enfermería, que hayan realizado prácticas clínicas y de forma voluntaria quisieran participar.

Se realizó la solicitud con las autoridades respectivas para poder convocar a los estudiantes, luego de su aprobación, se invitaron y se citó a los que participarían en una aula destinada para realizar las entrevistas.

Se inició con los estudiantes de $8^{\circ}$ semestre, luego fue $6^{\circ}$ y para finalizar $2^{\circ}$ semestre, se eligió este orden por motivo del calendario de las prácticas clínicas, el número de participantes fueron: 10 de $2^{\circ} ; 8$ de $6^{\circ}$ y 9 alumnos de $8^{\circ}$. En total fueron 27 participantes.

Después de contextualizar el estudio en cada grupo se solicitó la firma del consentimiento informado, así como la autorización para ser grabadas las sesiones; se explicó detalladamente la forma de participación del grupo focal y la función del investigador, que en este caso fue de moderar la participación de los estudiantes durante la entrevista.

Se espera que el investigador no interprete las participaciones de los informantes en ese momento, sin embargo, se asume que una de las funciones en el grupo focal es el desarrollo del grupo, por lo que se acepta que cuando hubo dudas conceptuales la moderadora intervino para clarificar y avanzar en el diálogo.

El tratamiento de los textos consistió en tres etapas: escucha, transcripción y replanteamiento de nuevas interrogantes; para la nueva entrevista focal, la saturación de la información se determinó con la segunda entrevista en la consideración de que se logró la homogeneidad en la diversidad y la intensidad de la información necesaria para el estudio.

La transcripción de las entrevistas fue a través del software Dragon Naturally Speaking 12, se cotejó la coherencia entre el audio y el texto y se codificaron de acuerdo con el siguiente orden; $A=$ alumno, \#=número de ubicación en la primera entrevista por alumno y número de acuerdo al semestre al que pertenece el estudiante, por ej.: A2-6. El análisis de las entrevistas se realizó a través de las etapas que propone Gadamer, en la primera, la empírica, se identificaron los textos relevantes, se le asignaron unidades temáticas, se agruparon por afinidad; la segunda, reflexiva o interpretativa, permitió analizar los textos, su 
coherencia, con el fin de estructurar oraciones claras, sin alterar el significado del texto, posteriormente se estructuraron las categorías y subcategorías.

Se realizó triangulación ${ }^{14}$, primero con los informantes, después con otros investigadores, con el fin de obtener mayor riqueza interpretativa y analítica, y con la teoría existente por el análisis de los datos bajo dos visiones teóricas, con el fin establecer su credibilidad. La objetividad de la misma se garantizó a través de la audiograbación de las entrevistas, la transcripción total mediante un software y la revisión de la coherencia, entre ellos. Así mismo, el análisis se realizó a través de una codificación abierta y posteriormente se verificó comparativamente con el software Altas TI 7, en él se establecieron los tipos de relaciones que guardan las categorías entre sí. Las relaciones identificadas resultaron similares de las dos maneras, por lo que se pudo alcanzar mayor certeza en la conceptualización de las categorías y subcategorías, que dan cuenta del fenómeno de estudio y su interpretación.

\section{Resultados}

Tras el análisis de los textos se identificaron cinco categorías generales que son las siguientes: Autopercepción de la Enfermería y las subcategorías: vocación de servicio, educación, holismo, integración de conocimientos, persona vs paciente; Arte del cuidado y las subcategorías: creatividad, desarrollo de la sensibilidad humana; Interacción enfermera-persona y las subcategorías: confianza, empatía, cultura, escucha activa; Conducta profesional y las subcategorías: dignidad humana, autoconocimiento/reconocimiento del otro y espiritualidad vs. religiosidad con las subcategorías: relación espiritualidad y arte, transcendencia, vida, muerte.

\section{Categoría 1. Autopercepción de la Enfermería}

La autopercepción es descubrir las emociones, deseos e ideas ${ }^{15}$ para formar una opinión sobre sí mismo o una situación que se vive, es el concepto propio de cada profesional, frente a lo que significa la enfermería, cómo la comprende, expresa y lleva a cabo desde la práctica y el desarrollo teórico, todo esto genera en la persona un autoconcepto de la profesión, que la convierte en propia y permite que se vuelva una forma de vida.

"La enfermería es el arte, la ciencia, la disciplina que se encarga de brindar cuidado, pero como apropiarnos de que enfermería... es un estilo de vida... para aquella persona que tenga esa vocación de servicio" A6-6.

Los estudiantes comprenden la enfermería como ciencia, arte y disciplina del cuidado que requiere de vocación de servicio; implica un querer cuidar con base en la necesidad del otro, es también un estilo de vida que integra conocimientos científicos, biológicos, psicológicos y espirituales, para la construcción de una visión holística de la persona, que se involucra en el cuidado a la familia a través de la comunicación e interacción permanente y la educación para la salud.

\section{Subcategoría 1.1. Vocación de servicio}

Para Martínez y Napione según refiere Francisco del Rey ${ }^{16}$, la vocación de servicio significa "darlo todo por el paciente”, es decir en el proceso de cuidado la enfermera da lo mejor de sí, tanto en conocimiento como éticamente para proporcionar los mejores cuidados. Del mismo modo, es un interés del propio ser a dedicar la vida, al cuidado de los otros.

"Un estilo de vida, naces como con esa vocación de querer ayudar a los demás... es poder ayudar a alguien de una manera desinteresada y que de todas maneras... las cosas salgan bien o salgan mal, te debes sentir a gusto y orgulloso de lo que haces" A1-6.

Los estudiantes refieren que la enfermería es una vocación de servicio, un don que no cualquiera tiene para dar cuidado a la persona, que se convierte en un estilo de vida que se da a partir de una ayuda desinteresada; este pensamiento se refleja más en los estudiantes de los semestres superiores, pues han tenido mayor contacto con las personas en prácticas, lo cual les permite una construcción de la identidad profesional de enfermería. 


\section{Subcategoría 1.2 Educación para la salud}

La educación para la salud involucra la promoción y prevención en el cuidado, dar una información certera para la persona y su familia sobre el proceso de salud- enfermedad que le permitirá hacer cambios en su estilo de vida.

En la Teoría de la trayectoria de la enfermedad ${ }^{17}$, la educación del sistema paciente-familia, considera todos los aspectos que lo rodean y busca reducir la incertidumbre que se presenta por la falta de información, que se tiene acerca del proceso de cuidado que tiene una persona.

"Informarle a la persona y al familiar lo que estamos haciendo para que conozca... está fundamentado y que haya confianza de las dos

partes... enseñar a la familia como cuidar en casa... depende de la situación en la que se encuentra el paciente” A6-6.

Se considera que la familia es el eje fundamental de cuidado a quienes se les debe enseñar cómo realizar los cuidados, para que la persona logre su bienestar y su autonomía, que no sólo depende de ella sino del apoyo de sus redes sociales. Se asume que la educación es un proceso permanente de interacción humana de enseñanza-aprendizaje, muy cercano a las características culturales y étnicas de la persona, familia y grupos.

\section{Subcategoría 1.3. Integración de conocimientos}

La formación en enfermería requiere de conocimientos teóricos, prácticos y humanísticos que permiten a los estudiantes adquirir un desarrollo profesional integral, durante el cual se verá reflejado el arte del cuidado.

Según Acebedo-Urdiales et $\mathrm{al}^{18}$, Benner refiere que el aprendizaje en los estudiantes integra tres aspectos: intelectuales, prácticos y éticos, que permiten desarrollar habilidades y capacidades del papel profesional de enfermería.

“Una combinación de... todos los conocimientos generales, científicos, biológicos, psicológicos que tenemos, más esa parte social, que tenemos que tener siempre presente, porque estamos tratando personas... no solamente es hacer procedimientos, es estar ahí para él, escucharlo, sabiendo sobre sus necesidades, sus preocupaciones, sus miedos, dándole la información, la información es poder y la información siempre nos quita el miedo" A7-6.

Los estudiantes consideran que la formación en enfermería debe ser integral, incorpora conocimientos humanísticos y teórico-científicos, para tener dominio de los procesos humanos donde pueda interactuar en una verdadera praxis y con ello dar un cuidado holístico.

\section{Subcategoría 1.4 Holismo}

La enfermería holística ${ }^{19}$ implica a la persona como un conjunto de subsistemas que se encuentran en interacción y que existe una relación entre la mente, cuerpo y espíritu, lo cual es importante conocer para la práctica del cuidado.

"Ver a la persona como un ser completo o algo como completo, ser humano, desde siempre hemos intentado entenderlo ya sea la parte anatómica, psicológica, social y demás, entonces yo siento que al hablar de holística nos estamos refiriendo todo ese ser, pero entenderlo de una forma completa que todo se va integrando" A12-2.

Desde el inicio de la formación se tiene una visión holística de la persona, es decir, como un ser completo, lo que implica una unidad compleja que se manifiesta ante la enfermera(ro) en un proceso de salud, enfermedad, sufrimiento o muerte.

\section{Subcategoría 1.5. Persona vs. Paciente}

Nightingale ${ }^{20}$, considera paciente a un sujeto pasivo, que se encuentra enfermo y en relación de cuidado controlada por la enfermera; mientras que Watson ${ }^{20}$ considera a la persona un ser de mente, cuerpo, espíritu y naturaleza con la capacidad de poder participar en su cuidado, tienen en cuenta el aspecto sociocultural.

"Yo considero que no es lo mismo... simplemente una persona somos nosotros, pero un paciente es el que está hospitalizado o internado... no va dejar de ser una persona, pero tiene, bueno se le pone una etiqueta... los pacientes, todos son personas, pero no son pacientes” A 1 1-2. 
Las palabras persona y paciente son utilizadas sin establecer aún una diferencia específica; aunque se considera paciente como una etiqueta o una persona en condiciones desfavorables, se puede usar según algunos estudiantes de igual manera sin distinción con persona, otros piensan que si existe una diferencia.

\section{Categoría 2. Arte del Cuidado}

Watson ${ }^{5}$ define arte de enfermería como la capacidad de un ser humano para percibir la expresión de los sentimientos a través de la escucha, mirada atenta y contacto físico.

"El concepto de arte es como expresar emociones, sentimientos, pero también lleva... todo un proceso, en enfermería hacemos algo... llevamos un seguimiento de todo" A12-2.

"Incluye esa parte de los sentimientos, de la manera en la que te vas a relacionar con las personas..., si lo vas a tratar como un paciente 0 como una persona igual a ti..., incluyendo los valores que se asemejan y los que tienes que implementar" A4-8.

Los estudiantes comprenden el arte en el plano emocional-espiritual, en donde la enfermera construye una intencionalidad cognoscitiva afectiva para el cuidado creativo, en una búsqueda de sentido y significado en el cuidado del otro, en una integración estética. Es una comprensión expresada en los discursos en todos los ciclos de formación, independientemente de las prácticas que hayan vivido.

\section{Subcategoría 2. 1. Creatividad}

La creatividad es esencial en el arte del cuidado, porque permite a la enfermera expresarse a través de intervenciones innovadoras que contribuyan a la búsqueda del bienestar de la persona.

Según Almansa ${ }^{22}$, la creatividad es una cualidad que permite a la persona solucionar cualquier situación vital, lo que apoya a la enfermera en la toma de nuevas decisiones o cambiar de estrategias para el próximo proceso de cuidado que realice.

“Podría ser hacer las cosas con fundamentación, hacerlas de una forma correcta..., poniendo parte de creatividad, empeño en que se pueda hacer mejor" A3-8.

La creatividad es la capacidad que tiene la enfermera de inventar algo nuevo, es decir, que se le facilite expresar de manera única su cuidado, igualmente puede adaptar el cuidado a las nuevas situaciones que esté viviendo la persona.

\section{Subcategoría 2.2. Desarrollo de la sensibilidad humana}

Para Pereira et al $^{23}$, la sensibilidad es una habilidad subjetiva que desarrolla el profesional a través de comportamientos y actitudes, que permiten a la persona cuidada expresar sus sentimientos y emociones, requiere de empatía, comprensión y amor por parte de la enfermera.

“Es como un sentimiento de utilidad o alegría, pero también a través de como... desarrollamos... la empatía, podemos llegar a sentir tristeza y miedo... sentir nervios... porque a veces no tengo los conocimientos suficientes” A6-6.

“Compasión, yo digo que hay que tenerla..., es complicado... yo he experimentado compasión, tristeza, también hasta cierto punto experimentar el mismo dolor que las personas están pasando y poder actuar” A5-8

Los estudiantes ante el contacto con la persona sentían miedo, seguridad, angustia, respeto y luego del cuidado alegría, compasión, tristeza, satisfacción por el trabajo bien hecho sin importar el desenlace de este cuidado, indica que hay una gran sensibilidad que se genera por el nivel de formación e inseguridad respecto a los conocimientos y otros de compasión y dolor por el otro, pero también amor y esperanza, sentimientos muy humanos empáticos con la persona que sufre, y se advierten como positivos en su identidad profesional.

\section{Categoría 3. Interacción Enfermera-Persona}

La interacción es una relación significativa entre la enfermera y la persona, que requiere de diferentes aspectos como son: la confianza, empatía donde el profesional se pone en situación comprensiva real para entender lo que está viviendo, la cultura que influye en su estado de salud, y por último, la escucha activa 
como un desarrollo de la voluntad de comprender lo que la persona expresa para concretar el proceso de comunicación; esta interacción va más allá de la mera relación enfermera-paciente tradicionalmente concebida.

\section{Subcategoría 3. 1. Confianza}

Para Torralba ${ }^{24}$ un elemento central en el arte del cuidado es la confianza, pues es ponerse en las manos de alguien más, creer en él y ponerse a su disposición; requiere de la enfermera no sólo de palabras sino de acciones y actitudes frente a la persona que se cuida, para generar seguridad en el proceso de cuidado.

"Mostrando seguridad de lo que haces, de lo que vas hacer, de lo que estás hablando... van a decir no pues esta enfermera si sabe, está hablando... del procedimiento que me va hacer y si te ve segura, va a confiar en ti” A5-2.

Los estudiantes refieren que la confianza es mostrar seguridad con el conocimiento adquirido ante la persona que se cuida, al expresar respeto, genera una comunicación autentica con el otro, donde no se critica o juzga, así se establece una confianza mutua que permita el proceso de cuidado.

\section{Subcategoría 3.2. Empatía}

Watson define la empatía como "la capacidad para la experiencia" ${ }^{5}$, es decir, permite a la enfermera comprender las sensaciones y emociones de la persona cuidada, esto favorece la comunicación y la confianza para realizar el proceso de cuidado.

“La empatía es ponerse en el lugar del otro... tratar de entender la situación que está pasando” A3-2.

“Ser asertiva, no tiene que perder el respeto y esa amabilidad, esa empatía por la otra persona, aunque sea fuerte la situación...” A1 0-2.

La empatía fue el término generalizado que los estudiantes utilizaron para expresar una actitud intencionada de comprensión del otro, es ponerse en el lugar del otro, con ello buscan entender la situación que está viviendo la persona en el momento, es poder encontrar la forma o las herramientas adecuadas para dar cuidado individualizado, es incorporar el conocimiento humano.

\section{Subcategoría 3.3. Cultura}

Según Noriega et $\mathrm{al}^{25}$, es el conjunto de los rasgos distintivos, espirituales, materiales, intelectuales y afectivos que caracterizan a una sociedad, se expresa en los modos de vida, los derechos fundamentales al ser humano, los sistemas de valores, las tradiciones y las creencias, que la cultura da al hombre la capacidad de reflexionar sobre sí mismo.

"Sus aspectos culturales y las costumbres... porque muchas veces en las estancias hospitalarias nos enfocamos a que todos necesitan cubrir las mismas necesidades y cada uno tiene una cultura, practicas diferentes a nosotros que en algún momento pueden llegar a no... estar de acuerdo con ello y... a ellos los identifica" A3-8.

Los seres humanos cuentan con un conjunto de creencias, valores y costumbres que se denomina cultura y lo identifica con un grupo social, estas influencian su forma de ver el entorno y su concepto de salud, enfermedad, así también como su espíritu; durante el proceso de cuidado la interacción enfermera-persona se ve influenciada por la cultura de las dos personas, puede afectar de manera positiva o negativa la forma de dar cuidado.

\section{Subcategoría 3.4. Escucha activa}

Subiela García et $a^{26}$ refieren que es la capacidad de comprender la situación de la persona cuidada, que expresa sus sentimientos y emociones a través de una comunicación verbal y no verbal, por ello escuchar es considerado un arte complejo del lenguaje.

"yo creo que escuchar sin interrumpir, no brindar juicios que se expresen... nosotros tener tacto ... para entender a la persona y así poder crear confianza" A4-6.

Escuchar es un acto voluntario que tiene una persona a través del cual comprende e interpreta la información dada por el otro, para responderla y utilizarla en una relación establecida, con el fin de 
generar un proceso de retroalimentación. Además, es el punto de partida en la comunicación, ayuda a la enfermera en el cuidado para entender al otro y establecer confianza.

\section{Categoría 4. Conducta Profesional}

Manera de comportamiento de la enfermera(ro) en donde se reflejan sus habilidades y capacidades profesionales, así como tener en cuenta la dignidad humana, lo que permite un autoconocimiento y reconocimiento del otro, a través del desarrollo de la sensibilidad humana.

\section{Subcategoría 4.1. Dignidad Humana}

Para Marín ${ }^{27}$ es una cualidad de una persona, con independencia y autonomía que tiene cuatro dimensiones: religiosa, ontológica, ética y social; involucra el derecho a la privacidad en todo el proceso de cuidado.

“Desde el momento en el que pides permiso para hacer lo más mínimo, ya empiezas a respetar lo que es la dignidad de la persona” A1-6.

"Pues es darle su lugar, saber que a pesar de que está bajo nuestro cuidado sigue siendo una persona con los mismos sentimientos que nosotros" A3-6.

Para los estudiantes, aunque al principio se generó confusión para definir la dignidad humana; aun así, se logró definirla como el valor de la persona que se respeta desde el momento en que inicia la interacción enfermera-persona.

\section{Subcategoría 4.2. Autoconocimiento/reconocimiento del otro.}

Para Guevara et al ${ }^{28}$ la enfermería implica el cuidar del otro, que es aceptar las virtudes y defectos de la persona que se cuida; pero también involucra un proceso de aprendizaje para la enfermera de saber escucharse a sí misma, trasmitir confianza, seguridad, afecto y sinceridad durante el proceso de cuidado.

"La persona es desde la especie un ser humano que ocupa diversas características... si yo me reconozco como persona puedo reconocer a los otros, reconocerme a mí como persona significa saber que estoy aquí para aprender de todo, que tengo sentimientos, que tengo emociones, experimento situaciones a lo largo de la vida” A5-8.

Los estudiantes refieren que para realizar el cuidado es necesario un autoconocimiento como persona-enfermera(o) y a partir de allí reconocer al otro como una persona integral, a la cual es necesario demostrarle un interés para que inicie la confianza, el respeto y pueda existir una verdadera interacción.

\section{Categoría 5. Espiritualidad Vs. Religiosidad}

Torralba $^{24}$ define a la espiritualidad como la capacidad de toda persona a reflexionar sobre sí misma, sus capacidades, cualidades, defectos y fortalezas, considerándola una cualidad universal; puede contribuir positiva o negativamente en el proceso de cuidado.

“Toda religión incluye espiritualidad, pero no toda espiritualidad incluye religión, la religión tiene que ver más con las creencias más concretas más allá de la propia persona, yo creo que la espiritualidad tiene que ver con el autoconocimiento junto con la autoaceptación, junto con la construcción del estatus interior, de quien soy y hacia dónde voy" A5-8.

La espiritualidad para los estudiantes, es un proceso de autoconocimiento, autoconcepto, que sintetiza los valores, las creencias de cada persona lo que permite afrontar situaciones difíciles en momentos trascendentes en la vida, en cambio la religiosidad es la creencia en un ser superior que proporciona paz, confianza en lo que se le pide y alivio emocional; pero se suele considerar en ocasiones lo mismo por lo que no se diferencian tácitamente en la vida cotidiana, aun para otorgar el cuidado.

\section{Subcategoría 5.1. Relación Espiritualidad y Arte}

Durante el proceso de cuidado al darse la interacción enfermera-persona se conoce a la persona, sus sentimientos y emociones, lo que permite dar un cuidado individualizado. 
“... yo siento que la espiritualidad es la forma en la que van a expresar ciertos miedos, ciertos temores, pero no van a estar como implícitos sino que tú los tienes que descifrar y en el arte suele ser lo mismo, hay cosas que no son explicitas sino solamente tú las sientes, las entiendes a tu manera, entonces es eso cada arte va a tener su propia esencia, cada persona va a tener su propia espiritualidad" A12-2.

El arte del cuidado se da necesariamente en la interacción enfermera-persona donde se expresan los sentimientos y emociones auténticos, se relaciona con la espiritualidad a través de la expresión de sentimientos, creencias, valores y costumbres, genera fortaleza y voluntad de seguir adelante, donde la persona reconoce la ayuda y la interacción se da de forma recíproca en una verdadera creación única.

\section{Subcategoría 5.2. Trascendencia}

Según Muñoz, et $\mathrm{al}^{28}$ la trascendencia es la esencia de una persona que le da sentido a la vida, los valores y creencias, lo que es la fuerza vital, la paz interior que se desarrolla con el contacto a la naturaleza, el arte, la música y las relaciones interpersonales.

“Te agradecían la parte de la amabilidad, en cuestión por ejemplo en salud colectiva, bueno comunidad... nuestro trabajo debe trascender más allá de la clínica o de un hospital, o sea, si no trasciende no estamos haciendo lo correcto" A12-2.

La transcendencia es dejar una huella en las personas a través de los cuidados que se le realizan, no solamente en el hospital sino en todos los ámbitos donde se puede dar cuidado. La vida trasciende cuando se vive auténticamente y permite una existencia mínimamente feliz.

\section{Subcategoría 5.3. Vida}

Según la filosofía ${ }^{30}$, es la totalidad que se desarrolla, resuelve y mantiene simple en un movimiento, determinada por una organización físico-química de la materia corpórea; la vitalidad de esta organización no basta, pues depende también de una naturaleza espiritual que permite el desarrollo armonioso del camino total.

"La vida es como una existencia funcional, o sea existes, pero tienes un papel que desempeñar o una función dentro del medio 0 algo así" A10-2.

Según los estudiantes la vida es una existencia funcional para desempeñar un papel en el mundo, una oportunidad de convivir con personas que inicia desde el momento del nacimiento, un momento indescriptible, donde se experimenta alegría, satisfacción, emoción y esperanza por parte de la enfermera que presencia ese instante.

\section{Subcategoría 5.4. Muerte}

Según la filosofía, es la relación especifica con la existencia humana, que puede ser considerado el inicio o el final de la vida, al igual que la posibilidad existencial, admite que existe una esencia que es inmortal como el alma que reencarna en un nuevo cuerpo o como una vida incorpore ${ }^{30}$.

"Es como un momento para la reconciliación para todos los sentidos... entonces si trabajar con las personas que están próximas a la muerte, pero también es importante que esta persona se vaya... en paz y tranquila, pero también ver como deja a las personas que siguen con vida, entonces es un proceso tanto de la persona como de sus allegados" A5-8.

La muerte es percibida como el final de la vida, una pérdida o algo natural, dejar este plano físico para transcender. Es buscar la tranquilidad a través de un momento de reconciliación para irse en paz y que no sólo se debe trabajar con la persona que va a morir sino con su familia también, pues son los que sufren la pérdida de su familiar y viven el proceso de duelo.

Todas estas categorías dan cuenta de los significados que subyacen en los estudiantes y que quedan al descubierto a través del análisis del discurso (Figura 1).

\section{Discusión}

Tras el análisis de los discursos, se encontró que para los estudiantes el arte del cuidado es comprendido como la relación que establece la (el) enfermera(o) con la persona cuidada, en donde se requiere por parte de enfermería habilidades y capacidades de comunicación, además de los conocimientos teóricos 


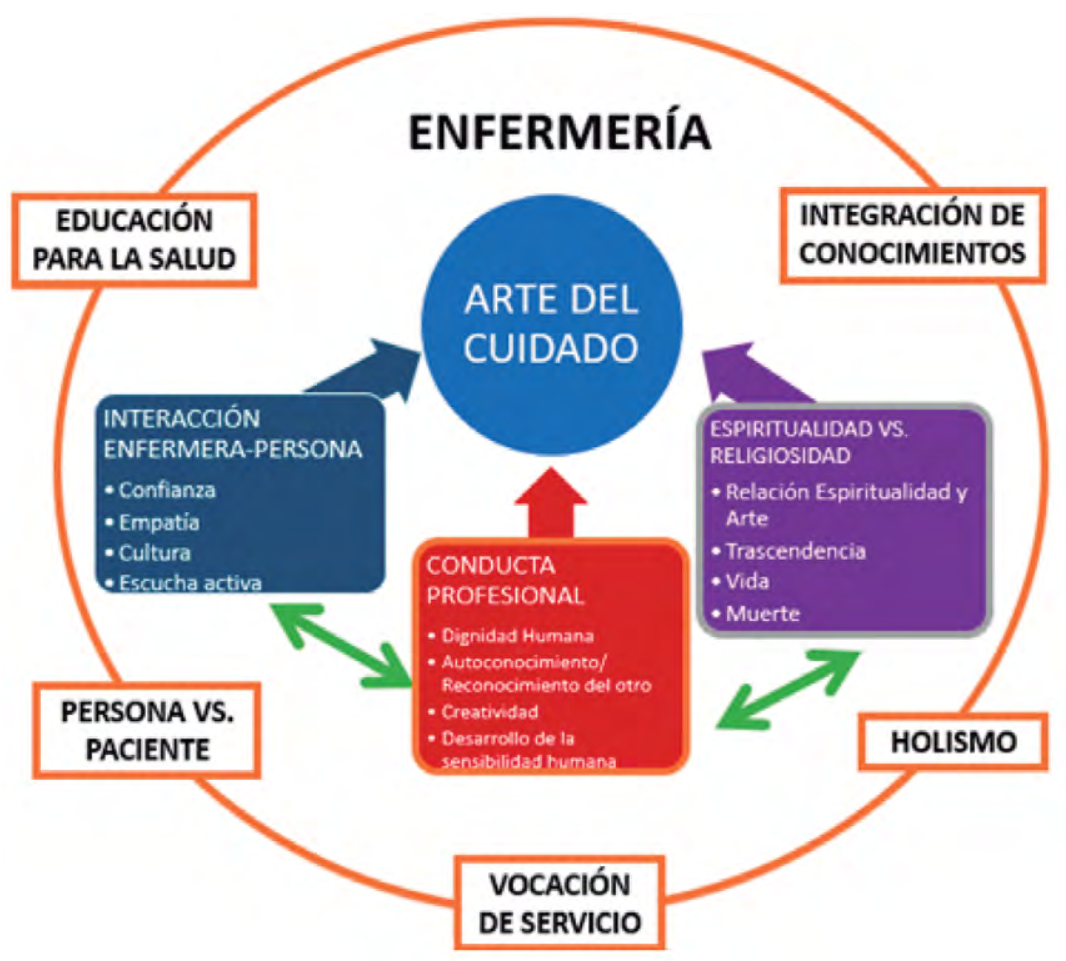

Figura I. Categorías de análisis identificadas y sus relaciones a partir del análisis de los textos de los grupos focales.

adquiridos en la formación. El proceso de autoconocimiento es uno de los significados del cuidado que le permite reconocer al otro como persona, desde una visión holística, donde se expresan los sentimientos, emociones y sensaciones tanto de la (el) enfermera(o) como de quien se cuida; esto se interpreta como la característica simultaneidad ${ }^{5}$ en el cuidado, que al mismo tiempo que se cuida se genera una fuente de salud recíproca, conceptos que Watson ha utilizado en su teoría del cuidado humano.

La categoría central identificada es la interacción enfermera-persona, dato empírico que se fortalece con el concepto del arte del cuidado de Carper ${ }^{29}$, comprende el acto de cuidar con un enfoque artístico en una interacción empática enfermera-persona que involucra compresión, autoconocimiento y observación; a través de una relación dialéctica, de igual forma que Muñoz-Pino ${ }^{10}$, quien reconoce que las emociones, sentimientos y sensaciones que expresa la persona ayuda a comprender y asimilar su situación, para disminuir la ansiedad y el temor mutuos.

Existe un aspecto que consideran como importante en el arte del cuidado y es la espiritualidad, como la expresión de los sentimientos, creencias, valores y costumbres de la persona, que le generan la fortaleza para afrontar la vida y los momentos difíciles. Lo que concuerda con el estudio de Magalhães ${ }^{11}$, quien apunta que los estudiantes entienden la dimensión espiritual como el apoyo, seguridad, confianza, orientación cuando la persona está angustiada y se busca dar consuelo.

Los significados que los estudiantes construyen en los procesos cognitivo-afectivo en su aprendizaje individual y colectivo, se nota mayor consolidación en los informantes de $2^{\circ}$ y $6^{\circ}$ en comparación con los de $8^{\circ}$ semestre; en los primeros, aún es vigente la información acerca de los fundamentos teóricos del cuidado y con respecto a los últimos, reciben mayor énfasis en la práctica dominante sobre los aspectos patológicos y procedimentales.

\section{Conclusiones}

Los significados que los estudiantes le otorgan al cuidado y al arte del cuidado se expresan a través de las categorías y subcategorías identificadas, en estas el arte del cuidado es una forma de vivir la enfermería en armonía con su ser profesional. 
Para los estudiantes el arte del cuidado significa desarrollo de la sensibilidad, creatividad en el cuidado a la salud, autoconocimiento, reconocimiento del otro en una relación de alteridad, respeto a la dignidad de la persona, desarrollo de la espiritualidad en el contexto de la conducta profesional.

Se destaca que la formación humanística se centra en cualificar al cuidado como holístico, con extensión a la familia, donde se reconocen los sentimientos implicados; hacen énfasis en el desarrollo de la identidad profesional-personal como componente esencial de la enfermería.

Se puede inferir que la formación humanística que expresan los estudiantes a través de los significados que le atribuyen al cuidado y al arte del cuidado, es de gran relevancia, ya que prestigian el compromiso personal y profesional como parte del desarrollo en su proceso formativo, se demuestra que poseen un marco de referencia teórico-filosófico humanístico caracterizado por los sentimientos, valores y actitudes que le asignan al profesional de enfermería.

Comprender la perspectiva de los estudiantes en el conocimiento de este fenómeno, resulta trascendente para reflexionar sobre el proceso formativo de enfermería, que implica a la docencia y a la investigación.

\section{Responsabilidades éticas}

Protección de personas y animales. Las autoras declaran que para esta investigación no se han realizado experimentos en seres humanos ni en animales.

Confidencialidad de los datos. Las autoras declaran que se protegieron los nombres de los participantes, estableciéndoles un código de referencia.

Derecho de privacidad y consentimiento informado. Las autoras declaran que previo a la obtención de la información los participantes leyeron y firmaron el consentimiento informado.

Financiamiento. Proyecto PAPIIT IN402915 "El Arte del Cuidado Enfermero desde la Visión de sus Profesionales en la Dimensión Estética".

Conflicto de intereses. Las autoras declaran no tener conflicto de intereses.

Agradecimientos. A los alumnos del $2^{\circ}, 6^{\circ}$ y $8^{\circ}$ semestre de la Escuela Nacional de Enfermería y Obstetricia, que participaron como informantes en el estudio.

\section{Referencias}

1. Escuela Nacional de Enfermería y Obstetricia. Licenciatura en enfermería. Organización curricular. CDMX: ENEO; 2016. [Consultado 14 septiembre 2015]. Disponible en: http://bit.ly/2deDAYg

2. Cárdenas-Becerril L. El Humanismo en la formación y práctica de enfermería: una esperanza transformadora. Texto contexto enferm. 2016; 25(1): 1-2.

http://dx.doi.org/10.1590/0104-070720160001.editorial

3. Pardo M, Núñez N. El humanismo como paradigma de la enfermería como ciencia. Av. Enferm. 1999; 17(1-2):22-30. [Consultado 22 de enero 2016]. Disponible en: http://bit.ly/2F7m2Ka

4. Johnson JL. A dialectical examination of nursing art. In: Cody WK. Philosophical and Teoretical Perpectives for Advanced Nursing Practice. $4^{\text {th }}$ ed. Burlington: Jones and Bartlett Learning; 2006. P. 131-42.

5. Watson J. Nursing: The philosophy and science of caring. Boston: Little Brown; 1979.

6. González-Juárez L, Velandia-Mora AN, Flores-Fernández V. Humanización del cuidado de enfermería. De la formación a la práctica clínica. Rev. Conamed. 2009; 14(Supl.1): 40-3. [Consultado 24 enero 2016]. Disponible en: http://bit.ly/2GdYJ2T

7. Garavito-Gómez A. La formación integral de estudiantes de enfermería. Investig. Enferm. Imagen. Desarr. 2013; 15(2): 153-72. [Consultado 25 enero 2016]. Disponible en: https://bit.ly/2zcxy5G

8. Parra A, Jiménez J, Olano J, et al. Primer acercamiento al paciente con cáncer: nuestra experiencia en el cuidado como estudiantes de enfermería. Invest. educ. enferm. 2005; 23(2): 148-52. [Consultado 3 septiembre 2015] Disponible en: http://bit.ly/2cB6wse 
9. Falcão IC, Lavínas MC, Mesquita GC. Arteterapia na assistência de enfermagem em oncologia: produções, expressões e sentidos entre pacientes e estudantes de graduação. Esc. Anna Nery $\quad$ 2007; 11 (2): 227-33. http://dx.doi.org/10.1590/S1414-81452007000200007

10. Muñoz-Pino IP. Experience of Nursing Students upon Their First Care Encounter with Terminally ill Patients. Invest. educ. enferm. 2014; 32(1): 87-96.

11. de Magalhães A, Limeira M, Braz C, et al. The Spiritual Dimension of Care in Nursing Practice: Student's Opinion. Journal Of Nursing UFPE / Revista De Enfermagem UFPE 2012; 6(9): 2037-44. https://dx.doi.org/10.5205/reuol.2570-20440-1-LE.0609201205

12. Soto-Nuñez CA, Vargas-Celis IE. La Fenomenología de Husserl y Heidegger. Cult. cuid. 2017; 21 (48): 43-50. http://dx.doi.org/10.14198/cuid.2017.48.05

13. Aguilar LA. La hermenéutica filosófica de Gadamer. Sinéctica. 2004; (24): 61-4. [Consultado 27 abril 2017] Disponible en: https://bit.ly/2KcU5Ux

14. Hernández-Sampieri R, Fernández-Collado C, Baptista-Lucio MP. Metodología de la investigación. $5^{\text {ta }}$ ed. CDMX: Mc Graw Hill Interamericana 2010. P.475-6.

15. Suriá-Martínez R. Intervención Psicosocial. Tema 3: Cognición y percepción social [Curso]. Alicante: Universidad de Alicante. 2010. [Consultado 15 marzo 2017]. Disponible en: http://bit.ly/2mugb7O

16. Francisco-del Rey JC. De la práctica de la enfermería a la teoría enfermera. Concepciones presentes en el ejercicio profesional [Tesis de doctorado]. Alcalá de Henares: Universidad de Alcalá; 2008.

17. Bailey DE, Stewart JL. Teoría de la incertidumbre frente a la enfermedad. En: Raile-Alligood M, Marriner-Tomey A. Modelos y teorías en enfermería. $8^{\mathrm{a}}$ ed. Barcelona: Elsevier; 2014. p.541-51.

18. Acebedo-Urdiales S, Rodero-Sánchez V, Vives-Relats, C, et al. La mirada de Watson, Parse y Benner para el análisis complejo y la buena práctica. Index Enferm 2007; 16(56): 40-44. [Consultado 26 abril 2016]. Disponible en: https://bit.ly/1MK0hBD

19. Hardin SR. Historia y filosofía de la ciencia. En: Raile-Alligood M, Marriner-Tomey A. Modelos y teorías en enfermería. $8^{a}$ ed. Barcelona: Elsevier; 2014. p.14-22.

20. Amaro MC. Florence Nightingale, la primera gran teórica de enfermería. Rev Cubana Enfermer. 2004 Dic [Consultado 6 octubre 2017] ; 20( 3). Disponible en: https://bit.ly/2PNJLba

21. Colchon K, Nuñez O. Cuidado Humano: Saber, Ser y Hacer del estudiante de Enfermería, según la teoría de Jean Watson en una Universidad Nacional de Lambayeque 2016 - 2017. [Tesis]. Perú: Universidad Nacional Pedro Gallo, 2018 [Consultado 6 enero 2018]. Disponible en: https://bit.ly/2F80oKH

22. Almansa P. Qué es el pensamiento creativo. Index Enferm. 2012; 21(3):165-8. http://dx.doi.org/10.4321/S1132-12962012000200012

23. PereiraA, Souza da Silva R, De Camargo CL, et al.Volviendo a los planteamientos de la atención sensible. Enferm. glob. 2012; 11(25): 343-55. http://dx.doi.org/10.4321/S1695-61412012000100021

24. Torralba F. Espiritualidad. Trad: Sala, R. España: Milenio. 2014.

25. Noriega JA, Carvajal CK, Grubits S. La Psicología Social y el concepto de cultura. Psicol. Soc. 2009; 21 (1): 100-7. [Consultado 26 agosto 2017]. Disponible en: https://bit.ly/2QrFqHQ

26. Subiela-García JA, Abellón-Ruíz J, Celdrán-Baños AI, et al. La importancia de la Escucha Activa en la intervención Enfermera. Enfermería glob. 2014; 13(34): 276-92. [Consultado 20 agosto 2017] Disponible en: http://bit.ly/2sgXDiE

27. Marín-Castán ML. La dignidad humana, los derechos humanos y los derechos constitucionales. Rev. Bioet. Derecho. 2007; (9): 8pp. [Consultado 20 agosto 2017]. Disponible en: https://bit.ly/2c13rE0

28. Guevara B, Zambrano A, Evies A. Cosmovisión en el cuidar de sí y cuidar del otro. Enferm. glob. 2011; 10 (21). [Consultado 22 agosto 2017] ; Disponible en: http://bit.ly/2vjs1Gz 
29. Muñoz A, Morales I, Bermejo JC, Galán JM. La Enfermería y los cuidados del sufrimiento espiritual. Index Enferm. 2014; 23( 3 ): 153-6. [Consultado 24 agosto 2017]. Disponible en: http://bit.ly/2vkPuYC

30. Abbagnano N. Diccionario de filosofía. 3 ed. FCE: México. 1998. p. 1188-9.

31. Carper B. Fundamental patterns of knowing in nursing. In: Cody W. Philosophical and Theoretical Perspectives for Advanced Nursing Practice. $4^{\text {th }}$ ed. Canada: Jones \& Bartlett Publishers; 2006. [Consultado 09 mayo 2016]. Disponible en: http://bit.ly/2cs6Wnd 